\title{
Multiple intron gain and loss events occurred during the evolution of Cenp-A gene
}

\author{
FAN XinYu ${ }^{1}, \mathrm{YU} \mathrm{Li}^{2,3}, \mathrm{XU}$ HuaiLiang ${ }^{1 *} \& \mathrm{LI} \mathrm{Ying}^{4 *}$ \\ ${ }^{1}$ College of Animal Science and Technology, Sichuan Agricultural University, Ya'an 625014, China; \\ ${ }^{2}$ Laboratory for Conservation and Utilization of Bio-resource, Yunnan University, Kunming 650091, China; \\ ${ }^{3}$ Key Laboratory for Animal Genetic Diversity and Evolution of High Education in Yunnan Province, Yunnan University, Kunming 650091, China; \\ ${ }^{4}$ Institute of Animal Breeding and Genetics, Sichuan Agricultural University, Ya'an 625014, China
}

Received July 27, 2012; accepted September 3, 2012; published online January 14, 2013

\begin{abstract}
Centromere protein A (CENP-A) is a histone H3 like protein, and it plays a very important role in chromosomal segregation during mitosis and meiosis. The analyses on the exon-intron organization of the Cenp-A gene in representative genomes revealed that multiple intron gain and loss events have occurred during the evolution of Cenp-A gene in opisthokonta (common ancestor of fungi and animals). Moreover, our results revealed that at least two positions were conserved in the intron gain and loss events during the evolution of the Cenp-A gene.
\end{abstract}

CENP-A, evolution, intron gain and loss

Citation: Fan X Y, Yu L, Xu H L, et al. Multiple intron gain and loss events occurred during the evolution of Cenp-A gene. Chin Sci Bull, 2013, 58: 2174-2178, doi: $10.1007 / \mathrm{s} 11434-012-5623-\mathrm{z}$

The centromere is composed of a tandemly repetitive satellite sequence and a protein complex [1]. More and more evidences have shown that centromere protein A (CENP-A) binding onto the centormeric region is an early step in kinetochore formation, although the process of the assembly of the complex is not clear [2]. CENP-A is a variant protein of histone H3 [3] containing a highly variant N-terminal tail, which diverges greatly in both the lengths and the amino acid compositions, while the C-terminal domain shares an average of $57 \%$ amino acid identities with histone $\mathrm{H} 3$ [4]. Histone $\mathrm{H} 3$ was replaced by CENP-A at the centromeric region of the necleosome [5]. The nearly invariant histone $\mathrm{H} 3$ has been maintained by a strong purifying selection during eukaryote evolution [6]. In contrast, Cenp-A evolved rapidly, especially in Drosophila [7,8] and Arabidopsis [9], where the rapid evolution is associated with positive selection [8].

Cenp- $A$ gene has been detected in all examined eukary-

*Corresponding authors (email: endlesssnow2012@gmail.com; huailxu@yahoo.com) otes [10]. In Drosophila, only one exon is identified, but in all mammals, birds and frogs, a 4 exon-3 intron organization (or gene structure) have been observed [11]. However, the gene structural evolution of Cenp-A is unclear. Here we compared the structure of Cenp-A gene in representative species from fungi to mammals and observed multiple intron gain and loss events in the Cenp-A gene during the eukaryotic evolution.

\section{Materials and methods}

The cDNA sequences of Cenp- $A$ gene from mammals (human, rhesus monkey, cattle, dog, mouse and rat), amphibians (frog), fishes (zebrafish, fugu, tetraodon), echinodermata (sea urchin), nematodes (C. elegant), insects (A. gambiae, D. meglanogaster, $S$. aegypti) and fungi ( $S$. cerevisiae) were downloaded from GenBank (the accession numbers are shown in Table 1). Here we only selected those species for which both the cDNA of Cenp-A and the genomic sequences 
Table 1 The cDNA sequences used and their chromosomal distribution of Cenp-A gene

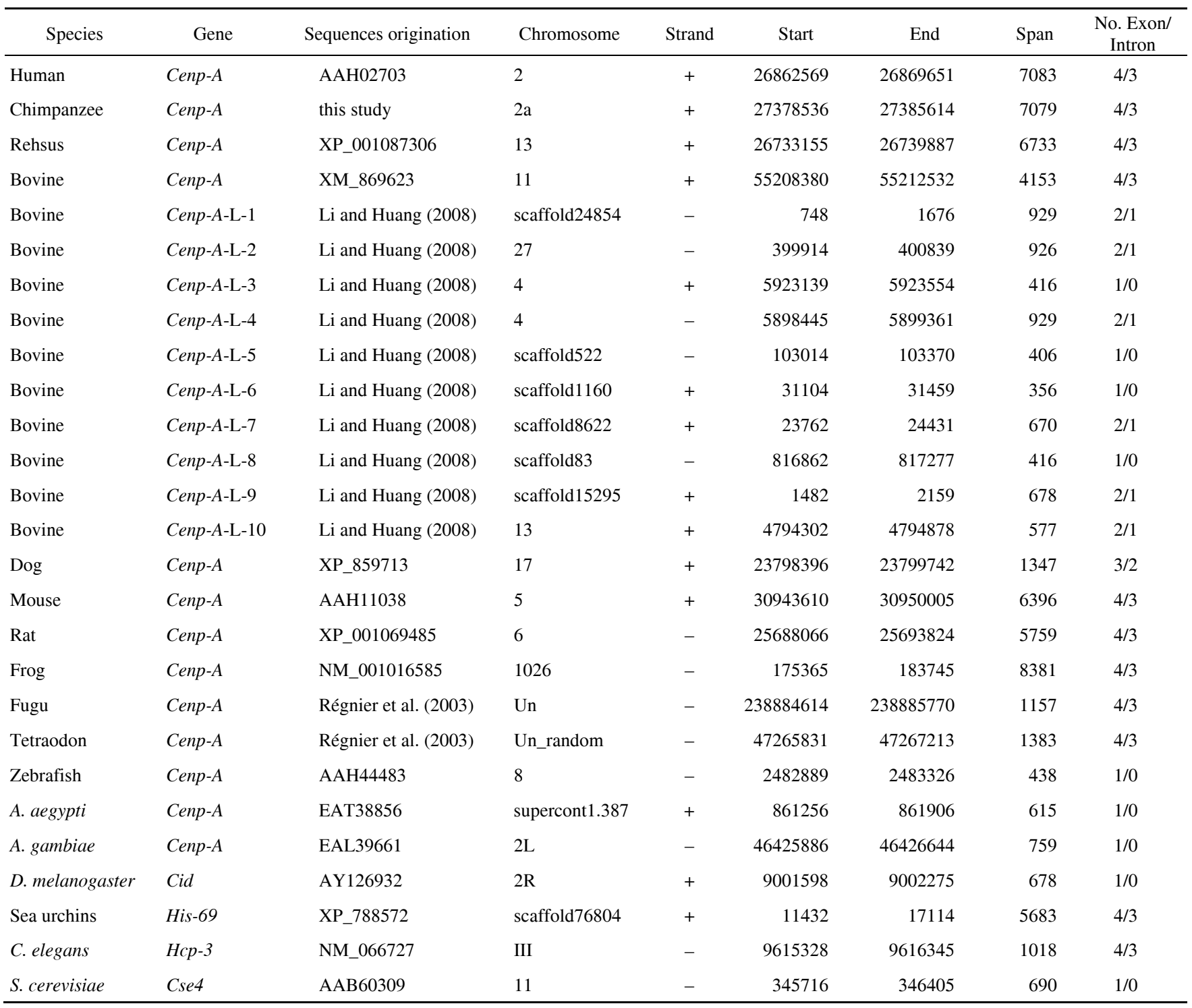

are available, because of the great divergence in both the amino acid composition and the length of the N-terminal region among different species, which makes it difficult to obtain the Cenp- $A$ gene from the genome sequence even by using a cDNA sequence from a slightly remote species as a query. We had no problem in extracting chimpanzee Cenp- $A$ coding sequences from its genomic sequence by using the human Cenp- $A$ as a query. The Cenp- $A$ gene sequences were extracted by mining their genome database (http://genome.cse.ucsc.edu/cgi-bin/hgBlat). And the obtained genomic DNA sequences and the cDNA sequences were used to conduct cDNA-to-genomic sequence alignment on Spidey (http://www.ncbi.nlm.nih.gov/IEB/Research/ Ostell/Spidey/), which provided the exon-intron structures. Repetitive elements in Cenp-A gene sequences were identified by the RepeatMasker program (http://www.repeat- masker.org/).

\section{Results}

\subsection{Gene structure evolution of Cenp-A}

The results from database searches show that some of Cenp- $A$ genes are intronless. The sizes of these genes and their locations in chromosomes are shown in Table 1, and the Cenp-A gene structural evolution is shown in Figure 1. Cenp-A genes in one fungus ( $S$. cerevisiae) and in three insects (A. gambiae, D. meglanogaster and S. aegypti) are intronless, which is also observed in all the 11 published Drosophila genomes. However, Cenp-A gene is interrupted by three introns in $C$. elegans and sea urchin (Figure 1). Most interestingly, different exon-intron structures are identified 


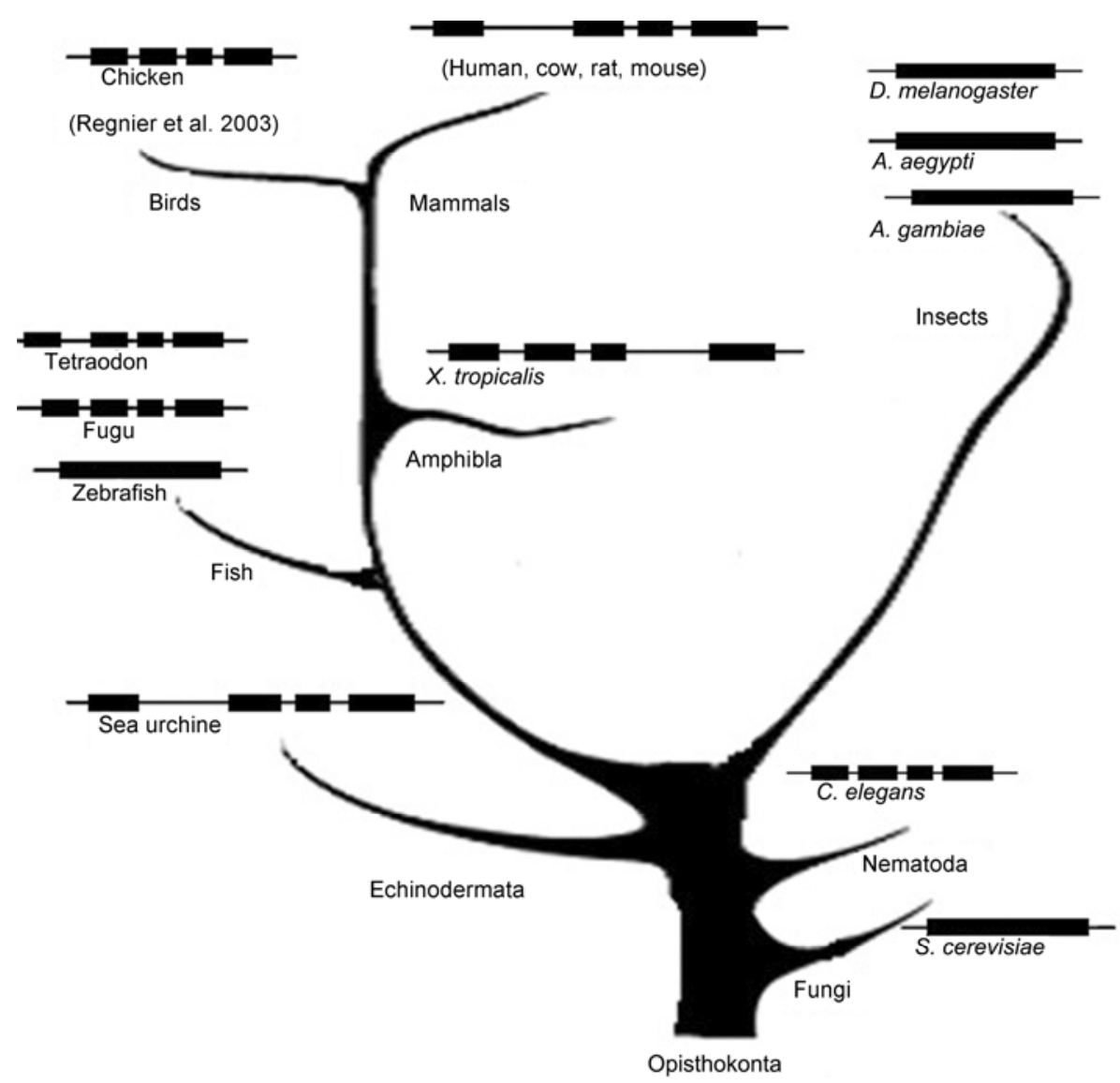

Figure 1 Scheme of the gene structure evolution of Cenp-A in opisthokonta. The tree is a simplified "tree of life" adapted from Eirin-Lopez et al. 2004, the intron-exon organizations are marked for those representative species, and the size of the lines (represent introns) and the boxes (represent exons) are not proportional to the lengths of the exon and the intron.

in fish, and three introns in fugu and in tetraodon are detected, but no intron is observed in zebrafish Cenp- $A$ gene. All Cenp- $A$ genes from amphibians, birds and mammals contain 4 exons and 3 introns except for that in cow, where a gene family with different exon-intron organizations among family members was observed [12]. We also proposed a most possible evolutionary relationship among different exon-intron forms (Figure 2 in [12]), in which we shown that only intron 2 was retained during the loss of introns form the original $4 / 3$ to $1 / 0$ structures [12]. Interestingly, the intron gain events have occurred at least twice: the first one occurred before the emergence of fungi and after the emergence of Nematoda (Figure 1); the second one occurred after the separation of the superorders Ostariophysi (including zebrafish, goldfish, and carp) and Acanthopterygii (including medaka, fugu, cichlid, etc.) [13]. And after the intron gain events, intron loss event has occurred at least once before the emergence of the insect. The positions and the phases (all phase 0) of intron 2 and intron 3 are conserved from sea urchin to mammals, in which the insertion site of the intron 2 in human is behind the 70th codon, and the insertion is behind the 96th codon for intron 3 , and no intron sliding (change of the intron position) is observed, thus, suggesting the Cenp- $A$ gene contains, at least, two hotspots of intron gain and loss.

\subsection{Distributions of the repetitive elements in Cenp- $A$ gene}

We observed that even for those species with the same 4 exon- 3 intron organizations of the Cenp- $A$ gene, the lengths of the gene diverged greatly from 1018 in C. elegans to 8381 in frog. What makes this great difference? Is repetitive sequences insertion a possible reason? To test this possibility, we checked the distribution of repetitive elements in those 4 exon-3 intron Cenp- $A$ genes. The results indicate that those short sequences (shorter than $2000 \mathrm{bp}$ ) contain no (or only one) matching repeats, while long sequences harbor more repetitive insertions, which differed both in the repeat types and their numbers. Table 2 shows the location and diversity of all repetitive elements found in the Cenp-A gene. We note that the divergence of the gene length is much less when those repeats are deleted; this is especially the case in mammals. The Cenp- $A$ gene length in mammals divergent from 2307 to 3864 when the repetitive elements were deleted, indicating that the great divergence of the gene length of Cenp-A due mainly to the insertion of the repetitive elements. 


\section{Discussion}

The study in the exon-intron organization of Cenp-A suggests that multiple intron gain and loss events occurred during the evolution of Cenp-A gene. These repeatedly occurred gain and loss of all the three introns (except for those in cow) is unexpected and erratic. More and more evidences have shown that introns are not "Junk DNA" as believed before. They may have functions, such as expression regulation [14], alternative splicing [15] and exon shuffling [16]. Although we cannot find any study to show direct evidence on whether or not any intron of Cenp-A has a functional effect, a research done by Osborn and Miller [17] showed that a intronless yeast CSE-4 (homologous gene of Cenp-A) can rescue a Cenp- $A$ knocked down human cell, which suggest that the functional effect of Cenp- $A$ introns if has, is not vital. This may further suggest that the multiple gain and loss of introns in Cenp-A might has just happened by chance and is evolutionarily neutral.

The intron density (number of introns per gene) is different among different genomes, so different tendencies of intron gain and loss are possible. The intron density from some representative species show that early branches are intron poor, while late branches are intron rich in the phylogeny of eukaryotic [18]. For example, birds and mammals have the values over 7 , that for $S$. cerevisiae is only 0.053 , but it is not always the case. For example, although $D$. melganogaster is higher than $C$. elegans in the phylogeny, its intron numbers per gene value is smaller than that in C. elegans. Most interestingly, the gene structure evolution of Cenp- $A$ seems consistent with the intron density described by Jeffares et al. [18]. In S. cerevisiae and D. molanogaster, the Cenp-A gene contains no intron, but 3 introns are observed in other species, and the intron density is relatively low. The Cenp-A gene in zebrafish is also intronless, but its intron density is unclear yet. Thus whether the intron loss is also related to lower intron density in this species is unclear. Because of the great variances of the N-terminal of the Cenp-A both in the amino acid composition and in its length, the attempt to acquire a Cenp- $A$ gene by searching its genome using a cDNA or amino acid sequences of Cenp-A from other taxon has failed. Thus the results in this paper may not reflect the whole gene structure evolution of Cenp-A. Even though, the results are still helpful for future studies to clarify how this gene origination changes occurred, and what are the factors that caused these changes.

The analyses on the distribution of the repetitive elements in the intron region of the Cenp-A gene show that the great gene length diversity of the Cenp-A genes was due mainly to the length differences of the repetitive elements in different species; this is especially the case in mammals. In several independent insertions, the most obvious example is occurred in amphibians and mammals, where the repetitive elements locate in intron 1 and intron 3 respectively (Table 2 ), so the insertion events is independent origin. And some linage specific repetitive elements have inserted into the Cenp- $A$ genes after mammals diverged from other vertebrates, for example, one ELVR insertion is primate specific, and the insertions of ALU/B1, B2-B6, ID3, MIRS and LTR are rodent specific. This independent insertion even occurred after the divergence of mouse and rat (Table 2), and thus suggesting species specific insertions. The insertions of repetitive elements in genome are evolutionary neutral in general, however, we believe it is also the case in Cenp-A introns, because (1) the intron gain and loss of Cenp-A among different species is consistent with the intron density differences among corresponding species; and (2) the gain and loss of introns itself might be neutral as we discussed before.

Table 2 Repetitive elements distribution in the Cenp-A genes

\begin{tabular}{|c|c|c|c|c|c|}
\hline Gene & Repeat elements & Location & Total repeats & Gene span & $\begin{array}{l}\text { Gene span after } \\
\text { deleted repeats }\end{array}$ \\
\hline Human Cenp-A & 9 SINE 6 LINE and 1ERVL & intron 1 & 4184 & 7083 & 2899 \\
\hline Chimpanzee Cenp-A & 9 SINE 6 LINE and 1ERVL & intron 1 & 4185 & 7079 & 2894 \\
\hline Rehsus Cenp-A & 8 SINE 6 LINE and 1ERVL & intron 1 & 3811 & 6733 & 2922 \\
\hline Bovine Cenp-A & 4 SINE 4 LINE & intron 1 & 1846 & 4153 & 2307 \\
\hline Rat Cenp-A & 12 SINE 1ALU/B1 7 B2-B6 3ID3 1 MIRS 1 LTR & intron 1 & 1895 & 5759 & 3864 \\
\hline Frog Cenp-A & 4 DNA transposons and 1 satellites & intron 3 & 1579 & 8381 & 6802 \\
\hline Fugu $C e n p-A$ & None & - & - & 1157 & - \\
\hline Tetraodon Cenp- $A$ & $1 \mathrm{LINE}$ & intron3 & 238 & 1383 & 1145 \\
\hline Sea urchins His-69 & None & - & - & 5683 & - \\
\hline
\end{tabular}


This work was supported by the National Natural Science Foundation of China (30970383), Program for New Century Excellent Talents in University $(N C E T)$.

1 Henikoff S, Ahmad K, Malik H S. The centromere paradox: Stable inheritance with rapidly evolving DNA. Science, 2001, 293: 10981102

2 Howman E V, Fowler K J, Newson A J, et al. Early disruption of centromeric chromatin organization in centromere protein A (Cenpa) null mice. Proc Natl Acad Sci USA, 2000, 97: 1148-1153

3 Palmer D K, O'Day K, Trong $\mathrm{H} \mathrm{L}$, et al. Purification of the centromere-specific protein Cenp-A and demonstration that it is a distinctive histone. Proc Natl Acad Sci USA, 1991, 88: 3734-3738

4 Sullivan K F. A solid foundation: Functional specialization of centromeric chromatin. Curr Opin Genet Dev, 2001, 11: 182-188

5 Warburton P E, Cooke C A, Bourassa S, et al. Immunolocalization of Cenp- $A$ suggests a distinct nucleosome structure at the inner kinetochore plate of active centromeres. Curr Biol, 1997, 7: 901-904

6 Piontkivska H, Rooney A P, Nei M. Purifying selection and birthand-death evolution in the histone $\mathrm{H} 4$ gene family. Mol Biol Evol, 2002, 19: 689-697

7 Malik H S, Henikoff S. Adaptive evolution of Cid, a centromerespecific histone in Drosophila. Genetics, 2001, 157: 1293-1298

8 Malik H S, Vermaak D, Henikoff S. Recurrent evolution of DNA- binding motifs in the Drosophila centromeric histone. Proc Natl Acad Sci USA, 2002, 99: 1449-1454

9 Talbert P B, Masuelli R, Tyagi A P, et al. Centromeric localization and adaptive evolution of an Arabidopsis histone H3 variant. Plant Cell, 2002, 14: 1053-1066

10 Warburton P E. Epigenetic analysis of kinetochore assembly on variant human centromeres. Trends Genet, 2001, 17: 243-247

11 Figueroa J, Pendon C, Valdivia M M. Molecular cloning and sequence analysis of hamster Cenp-A cDNA. BMC Genomics, 2002, 3: 11

$12 \mathrm{Li} \mathrm{Y}$, Huang $\mathrm{J}$ F. Identification and molecular evolution of cow Cenp-A gene family. Mamm Genome, 2008, 19: 139-143

13 Chen W J, Orti G, Meyer A. Novel evolutionary relationship among four fish model systems. Trends Genet, 2004, 20: 424-431

14 Chartier F L, Bossu J P, Vu-Dac N, et al. Involvement of intronic sequences in the transcriptional regulation of apolipoprotein $\mathrm{B}, \mathrm{E}$ and A-II genes. Z Gastroenterol, 1996, 34 Suppl 3: 44-45

15 Amy C M, Williams-Ahlf B, Naggert J, et al. Intron-exon organization of the gene for the multifunctional animal fatty acid synthase. Proc Natl Acad Sci USA, 1992, 89: 1105-1108

16 Bryk M, Belfort M. Spontaneous shuffling of domains between introns of phage T4. Nature, 1990, 346: 394-396

17 Osborn M J, Miller J R. Rescuing yeast mutants with human genes. Brief Funct Genomic Proteomic, 2007, 6: 104-111

18 Jeffares D C, Mourier T, Penny D. The biology of intron gain and loss. Trends Genet, 2006, 22: 16-22

Open Access This article is distributed under the terms of the Creative Commons Attribution License which permits any use, distribution, and reproduction in any medium, provided the original author(s) and source are credited. 